\title{
Crucial effect of system compliance on the maximum stress estimation in the hydrofracturing method: Theoretical considerations and field-test verification
}

\author{
Takatoshi Ito $^{1}$, Akira Igarashi ${ }^{1}$, Harumi Kato $^{2}$, Hisao Ito $^{3}$, and Osam Sano ${ }^{4}$ \\ ${ }^{1}$ Institute of Fluid Science, Tohoku University, 2-1-1 Katahira, Aoba-ku, Sendai 980-8577, Japan \\ ${ }^{2}$ Geotechnos Co. Ltd., 5-10-5, Shinbashi, Minato-ku, Tokyo 105-0004, Japan \\ ${ }^{3}$ Center for Deep Earth Exploration, Japan Agency for Marine-Earth Science and Technology, 3173-25 \\ Showa-machi, Kanazawaku, Yokohama, Kanagawa 236-0001, Japan \\ ${ }^{4}$ Earthquake Research Institute, University of Tokyo, 1-1-1 Yayoi, Bunkyo-ku, Tokyo 113-0032, Japan
}

(Received November 22, 2004; Revised April 12, 2005; Accepted May 4, 2006; Online published September 16, 2006)

\begin{abstract}
Why do the currently available data sets from field hydrofracturing tests indicate that the measured re-opening pressure lies close to the shut-in pressure far more often than can reasonably be expected? In order to explain such a strange phenomenon, it is necessary to take into consideration two additional factors, those of (1) a residual aperture of fracture and (2) hydraulic compliance of the test equipment, both of which are ignored in the conventional theory of hydraulic fracturing. The residual aperture causes pressure penetration into the fracture prior to opening, and its effect is to reduce the re-opening pressure by a factor of two from the value expected when the conventional theory is used. The conventional theory implies that the fracture always begins to open at a borehole pressure less than the shut-in pressure. However, due to the effect of large hydraulic compliance, the reopening pressure measured in the conventional manner becomes larger than the true reopening pressure and approaches the shut-in pressure. Contrary to this, the reopening pressure measured using the test equipment with sufficiently small compliance represents a good estimate of the true reopening pressure. This pressure is related to the maximum horizontal stress $S_{H}$, and its measured value allows us to estimate the value of $S_{H}$.
\end{abstract}

Key words: Stress measurement, hydrofracturing, maximum stress, reopening pressure, fracture opening, hydraulic compliance.

\section{Introduction}

It goes without saying that a knowledge of stress magnitudes and orientation is essential for understanding crustal dynamics. The stress at the depths under investigation can be measured from the results of in-situ tests carried out at those depths in boreholes. For the measurement at depths of more than $1 \mathrm{~km}$, hydraulic fracturing has been used generally since, compared with the other methods, the procedure and equipment involved in in-situ hydraulic fracturing are quite simple and appropriate for operating in such long and narrow spaces as boreholes.

Hydraulic fracturing in a vertical borehole induces fractures that will be vertical and normal to the minimum horizontal stress $S_{h}$ (parallel to the maximum horizontal stress $S_{H}$ ), if there is no influence of natural fractures. The fractures close with venting and open with re-pressurization. There appears to be two kinds of critical borehole pressure, i.e. the reopening pressure $P_{r}$ and the shut-in pressure $P_{s}$, both of which characterize the variation in borehole pressure during the test. The conventional theory tells us that those two pressures are related to the two stress components

Copyright (c) The Society of Geomagnetism and Earth, Planetary and Space Sciences (SGEPSS); The Seismological Society of Japan; The Volcanological Society of Japan; The Geodetic Society of Japan; The Japanese Society for Planetary Sciences; TERRAPUB. of $S_{H}$ and $S_{h}$ as follows:

$$
\begin{gathered}
P_{r}=3 S_{h}-S_{H}-P_{p}, \\
P_{s}=S_{h},
\end{gathered}
$$

where $P_{p}$ is pore pressure. These two equations give the principle for the two values of $S_{H}$ and $S_{h}$ to be determined from the two measured pressures of $P_{r}$ and $P_{s}$.

The interpretation of Eq. (2) for $P_{s}$ is supported by considerable experimental and theoretical investigations. On the other hand, if the interpretation of Eq. (1) for $P_{r}$ is also correct, the measured values of $P_{r}$ and $P_{s}$ should change independently in response to the combination of $S_{H}$ and $S_{h}$, which will vary site by site. However, the data obtained from field tests to date indicate that the measured re-opening pressure lies close to the shut-in pressure, i.e. $P_{r}=P_{s}$, far more often than can reasonably be expected (e.g. Evans et al., 1989; Lee and Haimson, 1989). While this unexpected and unusually situation could arise if the crust were in a stress condition of $\left(S_{H}-P_{p}\right) /\left(S_{h}-P_{p}\right)=2$, it is hard to accept that such a stress condition is continually present everywhere in the crust. It may be more reasonable to consider that, contrary to the conventional theory, the measured re-opening pressure does not coincide with the "true" re-opening pressure, i.e. the borehole pressure at which the fracture truly begins to open from its mouth at the borehole wall and that pressure takes the same value with 


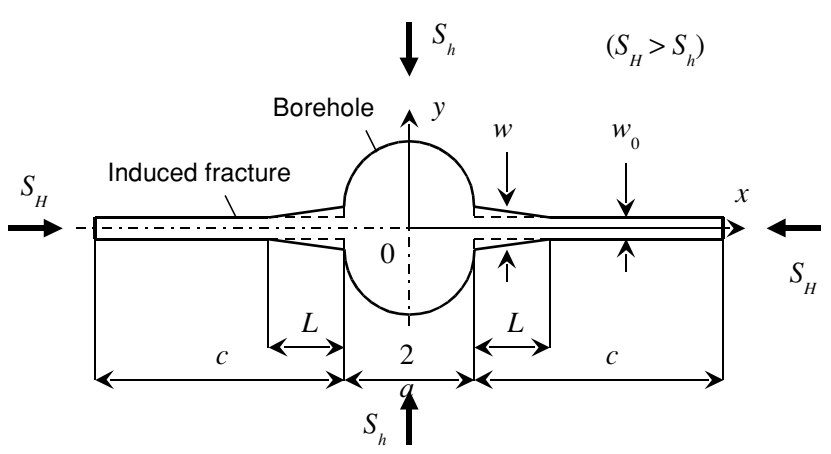

Fig. 1. Illustration of the fracture geometry used in the 2D numerical simulation of fracture-opening behavior. The fracture aperture $w$ is $w_{0}+w_{m}$, where $w_{0}$ is a residual aperture persisting when the fracture is closed, and $w_{m}$ is the additional opening caused by pressurization of the borehole and fracture. The length of the (additional) opened section at a given time is denoted by $L$.

the shut-in pressure, in other words with $S_{h}$. If this were true, hydraulic fracturing would only estimate the minimum component of stress, $S_{h}$, but not the maximum component of stress, $S_{H}$, which is the most desired parameter in the data sets on stress measurement. Furthermore, this large error in the estimates of maximum stress, $S_{H}$, based on the re-opening pressure may cause serious problems.

Taking account of these situations, in a previous work (Ito et al., 1999) we examined the relationship between the measured reopening pressure and in-situ stresses by means of numerical simulation. The results of this simulated showed that due to the effect of hydraulic compliance of the test equipments, the measured reopening pressure will become larger than the true reopening pressure and approach the shut-in pressure. Such an effect of hydraulic compliance on the measured reopening pressure has also been pointed out by Rutqvist et al. (2000). In the present work, we proceeded further with the numerical analysis to examine in more detail how a borehole pressure-time curve will change its shape according to fracture opening and the magnitude of $S_{H}$. We then conducted field experiments at the Kamaishi iron mine in the northeast district of Honshu, Japan's main island. We prepared two kinds of hydrofracturing system-i.e. compliant and stiff systems-and examined the effect of system compliance on the variation of borehole pressure by comparing the results of fracture opening tests using each system for the same fracture. Based upon these results, we were able to identify the conditions under which the measured reopening pressure represents a good estimate of the true reopening pressure. This pressure is related to $S_{H}$, and its measured value allows us to estimate the magnitude of $S_{H}$.

\section{Theoretical Considerations}

\subsection{True and apparent reopening pressures}

Equation (1) introduced by the conventional theory assumes that no pressure penetration of the fracture occurs prior to the onset of fracture opening. However, contrary to the assumption, as shown in laboratory investigations which have studied the permeability of fractures under compressive loads (e.g. Zoback et al., 1977; Cornet, 1982; Durham

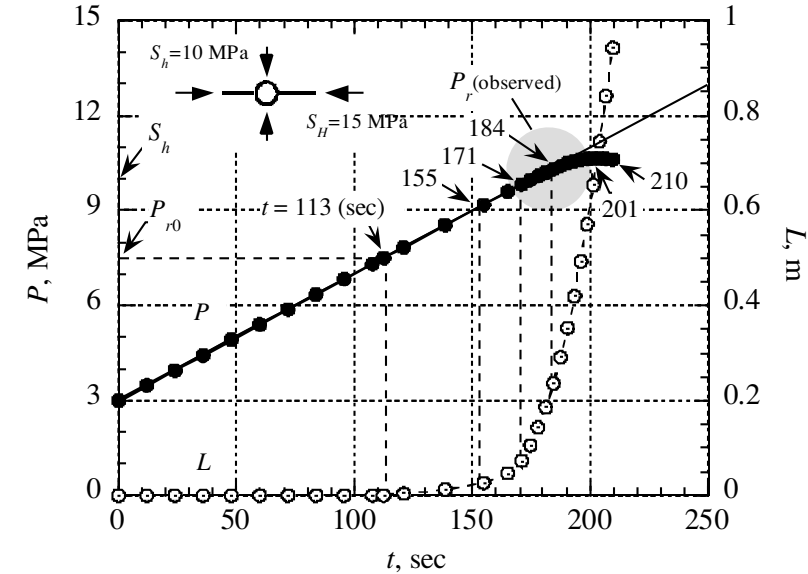

Fig. 2. Histories of borehole pressure, $P$, and fracture opening length, $L$, predicted by the numerical model illustrated in Fig. 1. The two fractures begin to open when the borehole pressure reaches $7.5 \mathrm{MPa}$, whereas the pressure shows no deviation from a linear trend until 10.3 MPa.

and Bonner, 1994), there are good grounds to believe that the retention of a residual aperture allows borehole pressure to penetrate the fracture before it begins to open. Based on this assumption and with the aid of numerical simulation, we examined how the residual aperture affects pressure distribution in the fracture and pressure variation in a borehole during the hydrofracturing test. Details on the numerical model have been reported in previous papers (Ito and Hayashi, 1994; Ito et al., 1999).

We assumed the induced fracture to be a 2D fracture, as illustrated in Fig. 1. Fluid flow in the fracture is approximated by laminar flow between smooth parallel plates of separation, $w$, and it is assumed that the induced fracture retains a residual aperture, $w_{0}$, when closed (i.e. when ambient conditions are restored after each injection cycle). Thus, the net fracture aperture $w$ is given by

$$
w(x, t)=w_{0}+w_{m}(x, t),
$$

where $w_{m}$ is the additional opening caused by fluid pressure in the borehole and fracture, $x$ is the coordinate axis parallel to the fracture line and $t$ is injection time. The opening behavior of fracture is simulated using a relation derived from fracture mechanics. Normal stiffness of the fracture is assumed here to be infinity so that the effect of fracture stiffness on the fracture opening is neglected. The rock is assumed to be impermeable so that poro-elastic stress and leak-off from the fracture face are ignored.

An example of a simulation is presented in Fig. 2, where we show the histories of borehole pressure, $P$, and instantaneous open fracture length, $L$, which is predicted for a constant rate injection cycle (i.e. note that $L$ is the length of the section where $w_{m}>0$ in Fig. 1). The parameter values used in the simulation are described below:

$$
\begin{aligned}
& S_{H}=15(\mathrm{MPa}), S_{h}=10(\mathrm{MPa}), P_{0}=3(\mathrm{MPa}), \\
& Q=2 \times 10^{-6}\left(\mathrm{~m}^{3} / \mathrm{sec}\right)(=120 \mathrm{ml} / \mathrm{min}), a=0.05 \\
& (\mathrm{~m}), c=1(\mathrm{~m}), h=1(\mathrm{~m}), w_{0}=2(\mu \mathrm{m}), G=25 \\
& (\mathrm{GPa}), v=0.2, \mu=10^{-3}(\mathrm{~Pa} \mathrm{sec}), \beta=0.5 \\
& \left(\mathrm{GPa}^{-1}\right), C=5 \times 10^{-5}\left(\mathrm{~m}^{3} / \mathrm{MPa}\right),
\end{aligned}
$$

where $P_{0}$ is the initial value of borehole pressure (it may 


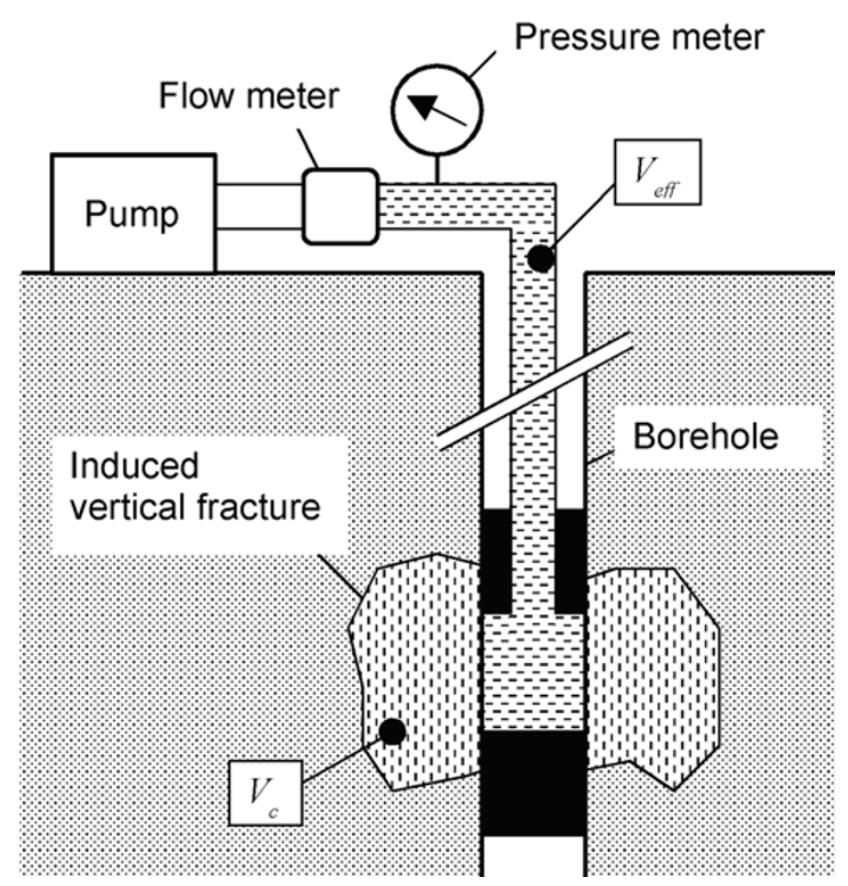

Fig. 3. Illustration of a typical hydraulic fracturing system showing the volume, $V_{\text {eff }}$, between the flow meter and the fracture mouths which give rise to wellbore storage both through fluid compressibility and volume change. The fracture volume is denoted as $V_{c}$.

be the pore pressure), $Q$ is the injection flow rate (taken as constant), $a$ is the borehole radius, $c$ is total fracture length (Fig. 1), $h$ is the fracture height, $G$ and $v$ are the shear modulus and Poisson's ratio of rock, respectively, and $\mu$ and $\beta$ are the viscosity and compressibility of the fracturing fluid, respectively. Note that the presence of a residual aperture, $w_{0}$, means that the fracture is filled with fluid at pressure $P_{0}$ from the beginning of pressurization. The effect of wellbore storage between the fracture mouth and the flow meter are described by the system compliance, $C$, or equivalently, the effective system volume, $V_{\text {eff }}$ (see Fig. 3), where $C=\beta V_{\text {eff. }}$ The compliance value used in the calculation is appropriate for conventional slim-hole (75$100 \mathrm{~mm}$ ) hydrofracture systems operating at depths of 100 $\mathrm{m}$ or less.

The history of borehole pressure in Fig. 2 shows that the pressure $P$ increases linearly with time until the pressure reaches $10.3 \mathrm{MPa}$, at which point it begins to deviate from the initial linear trend. Thus, in this example, the reopening pressure that would be measured by a pressure sensor in the test interval would be $10.3 \mathrm{MPa}$. This value is close to the $S_{h}$ of $10 \mathrm{MPa}$ but also not far off the value of the reopening pressure $P_{r}$ of $12 \mathrm{MPa}$ which is predicted by Eq. (1) taking account $P_{p}=P_{0}=3(\mathrm{MPa})$ in this case. However, the history of open fracture length in Fig. 2 shows that the fracture begins to open at $7.5 \mathrm{MPa}$. These results suggest that the deviation of the $P$ - $t$ curve from the initial linear trend occurs not only with fracture opening but also with other factors. To consider the reason for the discrepancy between the apparent (or measured) reopening pressure and the true reopening pressure, i.e. 10.3 and $7.5 \mathrm{MPa}$, respectively, in the example of Fig. 2, we made the plots of pressure $(p)$ and aperture $(w)$ distribution along the frac-
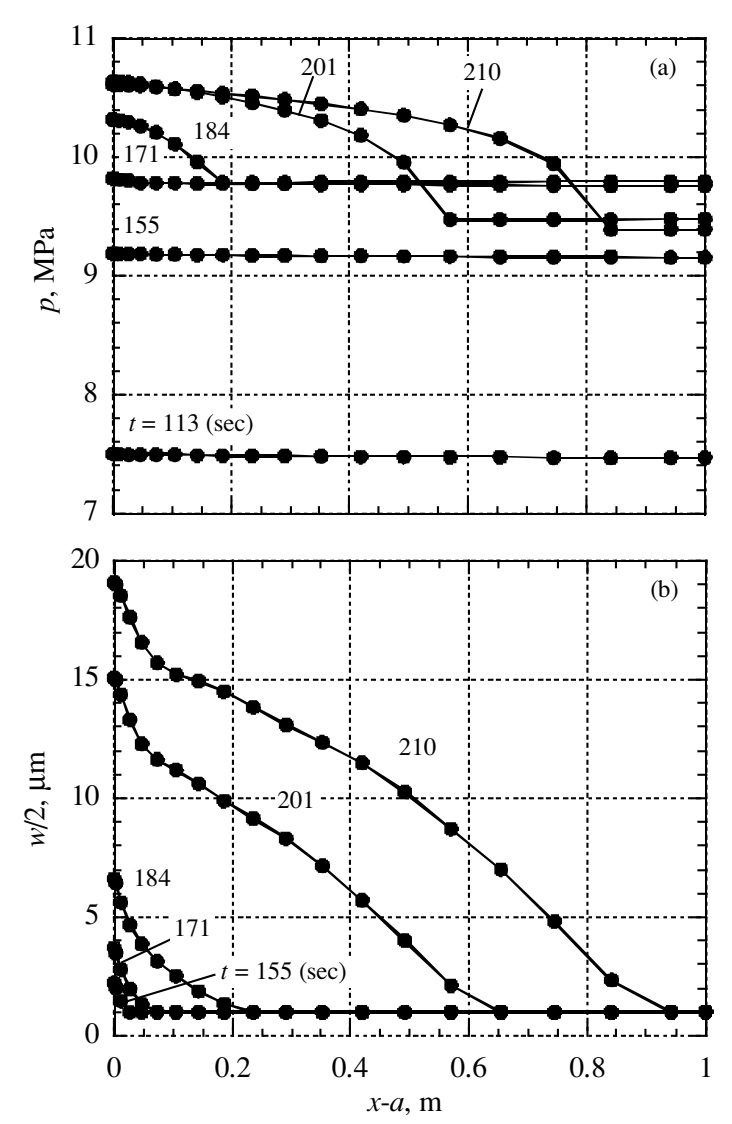

Fig. 4. Distribution of pressure $p$ (a) and aperture $w$ (b) along the fracture as a function of time $t$. Each line shows the result at the given time indicated beside the line, and the time is corresponding to that indicated in Fig. 2.

ture. The results are summarized in Fig. 4. Figure 4(a) shows that, despite assuming a small residual aperture of 2 $\mu \mathrm{m}$, there is significant pressure penetration into the fracture. The pressure distributes uniformly to be almost equal to the borehole pressure that is already present at the moment of fracture opening, i.e. $t=113$ (sec) (see Fig. 2). This result could be explained as follows. Even though the fracture volume is fixed until the fracture begins to open, an amount of fluid flows into the fracture from the borehole due to the effect of fluid compressibility. Nevertheless, because of the small residual volume of fracture, the amount of flowing fluid will be so limited that the pressure gradient in the fracture will be negligible and the fracture pressure will be elevated, thereby keeping its uniform distribution; this is also true for the result for $t=113$ (sec) in Fig. 4(a) with increasing borehole pressure. Taking account of this, the third component of Eq. (1), which is the pore pressure inside the fracture mouth, should be replaced by the borehole pressure $P$ instead of the pore pressure $P_{p}$. Of course, the $P$ at the onset of fracture opening is defined as the reopening pressure $P_{r}$, and replacing $P_{p}$ with $P_{r}$ in Eq. (1) results in the following equation.

$$
P_{r}=\frac{1}{2}\left(3 S_{h}-S_{H}\right) .
$$

This shows that the fracture begins to open at 7.5 MPa, a result which is consistent with the result of Fig. 2. Thus, 


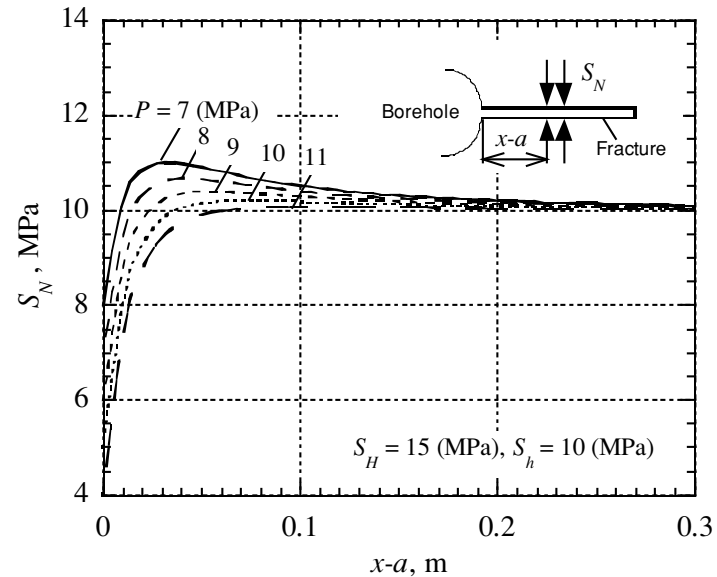

Fig. 5. Distribution of normal stress $S_{N}$ along the fracture and its variation with borehole pressure $P$, which is estimated from Eq. (6). With increasing $P$, the compressive stress concentration around the borehole is weakened; it finally almost disappears when $P$ reaches the value of the minimum stress $S_{h}$.

the effect of including pressure penetration into the fracture prior to opening is a reduction in the reopening pressure by a factor of two from the value expected using the conventional theory; this be seen comparing Eq. (1) (assuming $P_{p}=0$ ) and Eq. (4). Hereafter, we will denote the true reopening pressure and the apparent (or measured) reopening pressures as $P_{r 0}$ and $P_{r}^{(a)}$, respectively. $P_{r 0}$ is $7.5 \mathrm{MPa}$ and $P_{r}^{(a)}$ is $10.3 \mathrm{MPa}$ in the example of Fig. 2.

After the borehole pressure is beyond $P_{r 0}$, the fracture begins to open. Figures 2 and 4 show that, at the early stage, the fracture aperture increases slightly, while the borehole pressure increases considerably. Then, at the time around $t=184(\mathrm{sec})$, the fracture aperture increases abruptly with small increments in borehole pressure; at the same time the $P$ - $t$ curve begins to deviate from the initial linear trend. Assuming a negligible pressure gradient in the fracture, the effect of fracture opening on the borehole pressure variation can be expressed as follows (Ito et al., 1999):

$$
\frac{d P}{d t}=\frac{Q}{\frac{d V_{c}}{d P}+C}
$$

where $d V_{c}$ is the change in pressurized fluid volume due to fracture opening. Note that the compliance $C$ is defined as that of the volume between the fracture mouth at the borehole wall and the flow meter which is installed to measure the value of $Q$ (see Fig. 3). Since the flow rate $Q$ and the system compliance $C$ are constant, Eq. (5) indicates that deviations in the $P$ - $t$ curve from linearity are governed by changes in the value of $d V c / d P$ and its relative value with respect to $C$. That is, prior to fracture opening, $d V c / d P$ is zero and the borehole pressure $P$ increases linearly with $t$. After fracture opening, $d V c / d P$ becomes greater than zero, and the $P$ - $t$ curve will deviate from linearity to some degree. However, in the example of Fig. 2, the assumed value of $C$ is possibly so large compared with $d V c / d P$ at the early stage of fracture opening that no detectable change occurs on the $P$ - $t$ curve until $d V c / d P$ increases abruptly at $t=184(\mathrm{sec})$ and $P=10.3(\mathrm{MPa})\left(=P_{r}^{(a)}\right)$.
The reason why such an abrupt increase in $d V_{c} / d P$ occurs can be understood from the force balance at the fracture surfaces. The in-situ stresses $S_{H}$ and $S_{h}$ and the borehole pressure $P$ induce the stress acting normally to the fracture surface, $S_{N}$, which is given by

$S_{N}=\frac{S_{H}+S_{h}}{2}\left(1+\frac{a^{2}}{x^{2}}\right)-\frac{S_{H}-S_{h}}{2}\left(1+3 \frac{a^{4}}{x^{4}}\right)-P \frac{a^{2}}{x^{2}}$.

For the example of Fig. 2, this equation predicts the distribution of $S_{N}$ along the fracture line and its dependency on the borehole pressure, as summarized in Fig. 5. This result shows that, as long as the borehole pressure $P$ is smaller than the value of $S_{h}(=10 \mathrm{MPa})$, the fracture surfaces are compressed by the stress $S_{N}$ with a value larger than $P$ except in the vicinity of borehole wall. This means that the open portion of fracture is limited in the vicinity of the borehole wall, even if fluid pressure equal to the borehole pressure distributes uniformly inside the fracture and it pushes the fracture surfaces against $S_{N}$. Following this reasoning, the fracture aperture hardly increases as long as $P$ is less than the value of $S_{h}$. Then, with increasing $P$, the stress concentration in compression is weakened gradually due to the effect of the third term on the right side of Eq. (6) and, accordingly, the open portion of fracture becomes larger. When $P$ reaches a level of $S_{h}$, the stress $S_{N}$ becomes almost equal to or less than the value of $S_{h}$ anywhere. Such a balanced stress condition leads to the criticality that the fracture aperture increases abruptly with small increments in borehole pressure. As a result, $d V_{c} / d P$ becomes of a considerable value compared with $C\left(=5 \times 10^{-5} \mathrm{~m}^{3} / \mathrm{MPa}\right)$ and, finally, the $P$ - $t$ curve begins to deviate from the initial linear trend. The same process occurs regardless of the $S_{H}$ value (cf. Fig. 7(a)). Thus, this process provides an explanation as to why there are so many incidences where the apparent reopening pressure coincides with the shutin pressure - in other words, why situations of minimum stress $S_{h}$ are so common in the literature (e.g. Evans et al., 1989; Lee and Haimson, 1989). Note that considerable discrepancy between $P_{r}^{(a)}$ and $P_{r 0}$ does exist even though the assumption is made of an unlikely small value of the injection rate $Q$-i.e. $Q=2 \times 10^{-6}\left(\mathrm{~m}^{3} / \mathrm{sec}\right)(=120 \mathrm{ml} / \mathrm{min})$ in the example of Fig. 2. This result shows that $Q$ is not the key factor underlying the discrepancy between $P_{r}^{(a)}$ and $P_{r 0}$, while the discrepancy will become larger than in the case of Fig. 2 with increasing $Q$, as has been shown in Ito et al. (1999).

\subsection{Variation with system compliance}

The preceding analysis indicates that the discrepancy between $P_{r}^{(a)}$ and $P_{r 0}$ arises because the apparent system volume $V_{\text {eff }}(=C / \beta)$ of typical hydraulic fracturing systems is far larger than the fluid volume that enters the fracture during the initial stages of the fracture opening. This suggests that if $V_{\text {eff }}$, or its equivalent, system compliance can be made small enough, then $P_{r}^{(a)}$ may approach $P_{r 0}$, thereby allowing us to obtain useful estimates of $P_{r 0}$. We evaluated this possibility using a numerical simulator with the fracture-borehole geometry shown in Fig. 1. The parameter values used in the simulations are the same as those listed previously, except for $C$ and $S_{H}$. 

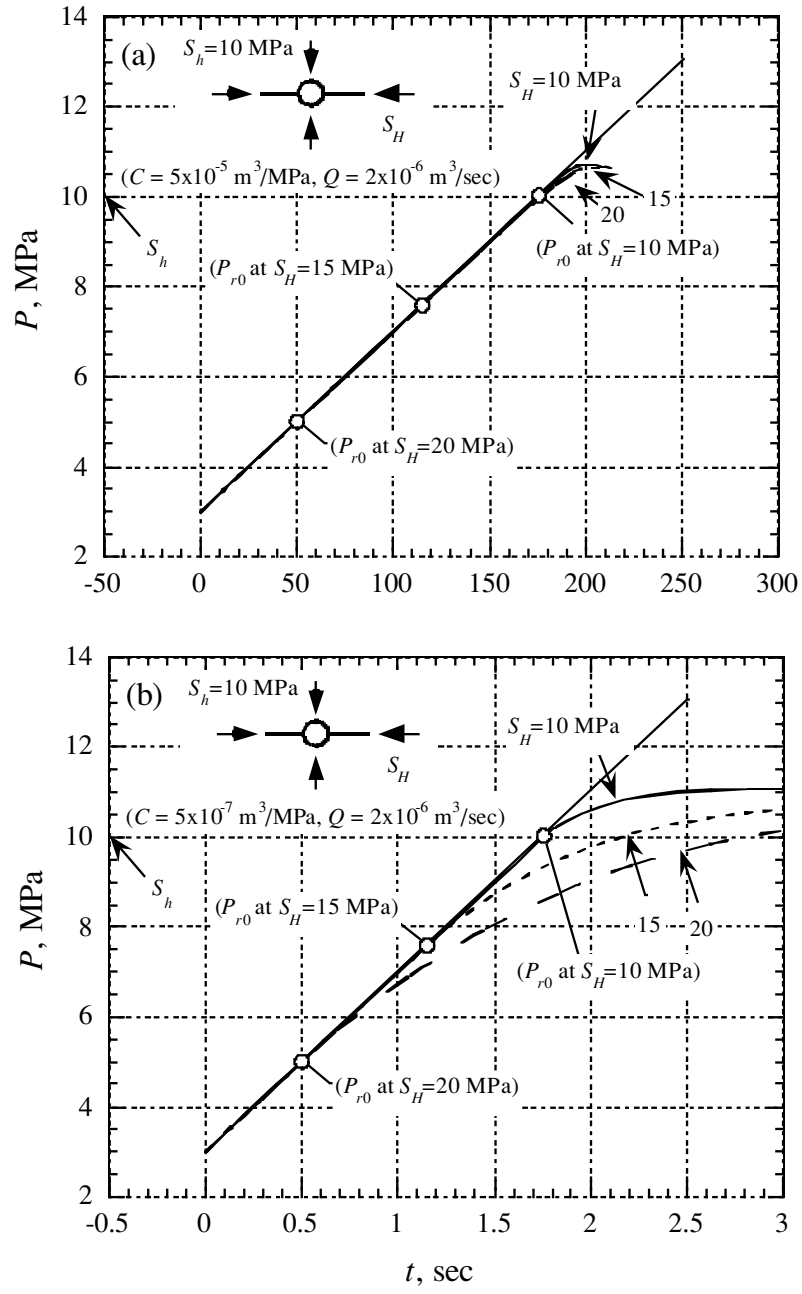

Fig. 6. Numerically predicted histories of borehole pressure and their dependency on the maximum stress $S_{H}$ for the cases using compliant (a) and stiff (b) hydrofracturing systems.

Examples of pressure-time curves obtained from the simulations assuming relatively large and small system compliances are shown in Figs. 6(a) and (b), respectively. In each figure, there are three curves obtained in which an $S_{H}$ of 10,15 and $20 \mathrm{MPa}$ is assumed, respectively. For the case assuming the relatively compliant system with $C$ of $5 \times 10^{-5} \mathrm{~m}^{3} / \mathrm{MPa}$ (see Fig. 6(a)), those three $P$ - $t$ curves are completely overlapped, and each apparent reopening pressure $P_{r}^{(a)}$ is significantly higher than $P_{r 0}$ and almost equal to the minimum stress $S_{h}$ of $10 \mathrm{MPa}$. In contrast, for the case assuming the relatively stiff system with $C$ of $5 \times 10^{-7}$ $\mathrm{m}^{3} / \mathrm{MPa}$ (see Fig. $6(\mathrm{~b})$ ), the $P_{r}^{(a)}$ coincides closely with $P_{r 0}$ for each case. Namely, for the cases assuming $S_{H}$ of 10,15 and $20 \mathrm{MPa}$, the $P_{r}^{(a)}$ are detected as $10.3,8.1$ and $5.9 \mathrm{MPa}$, and these values are close to $P_{r 0}$ of $10,7.5$ and $5 \mathrm{MPa}$ respectively. This result suggests that we could measure $P_{r 0}$ approximately from $P_{r}^{(a)}$ by using the relatively stiff system for fracture opening tests. The detected $P_{r}^{(a)}$ will allow us to estimate $S_{H}$ from Eq. (4) on the condition that the magnitude of $S_{h}$ is known from the shut-in pressure detected in some way. If this were the case, the value of $S_{H}$ is given by

$$
S_{H}=3 S_{h}-2 P_{r}^{(a)}
$$

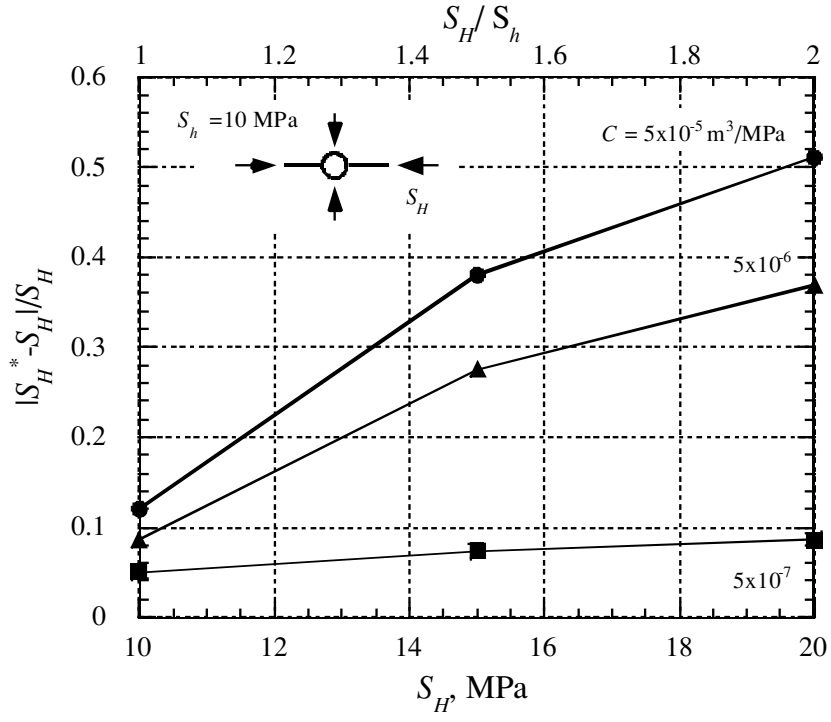

Fig. 7. Error in the estimates of maximum horizontal stress $S_{H}$ arising from the failure to measure the correct reopening pressure due to system compliance. Eq. (7) is assumed to be applied for the estimation. The error is reduced with reducing the system compliance $C$, and such a change occurs more drastically as $S_{H}$ becomes larger.

In the example of Fig. 6(b), Eq. (7) and a detected $P_{r}^{(a)}$ of $10.3,8.1$ and $5.9 \mathrm{MPa}$, the estimates of $S_{H}$ are 9.4, 13.8 and 18.2 , respectively, and these values coincide with the respective actual values of 10,15 and $20 \mathrm{MPa}$ within the error of $10 \%$. In the same way, we examined how such an error in the estimates of $S_{H}$ changes with system compliance. The results are summarized in Fig. 7. The error is plotted as a function of the true value of $S_{H}$, where $S_{H}$ * denotes the value of $S_{H}$ estimated by using Eq. (7) and the detected $P_{r}^{(a)}$. The scale of the stress ratio $S_{H} / S_{h}$ is also plotted in abscissa for comparison. The stress ratio is in the range of 1 and 2 and corresponds to $S_{H}$ in the range of 10 and $20 \mathrm{MPa}$, since the value of $S_{h}$ is fixed here at $10 \mathrm{MPa}$. Figure 7 shows that the error in $S_{H}$ * increases up to $50 \%$ of the true $S_{H}$ with increasing $S_{H}$, if system compliance is relatively large. The error declines with reducing system compliance and reaches the level less than $10 \%$ for a compliance of $5 \times 10^{-7} \mathrm{~m}^{3} / \mathrm{MPa}$. Note that $P_{r 0}$ approaches the value of $S_{h}$ as $S_{H}$ approaches $S_{h}$, and the pressure becomes exactly the same value of $S_{h}$ in the case of $S_{H}=S_{h}$. This implies that $P_{r}^{(a)}$ becomes close to $P_{r 0}$ irregardless of system compliance in the case of $S_{H}=S_{h}$. For this reason, the error in $S_{H} *$ for the case using compliant system decreases and approaches the error for the case using the stiff system as $S_{H}$ approaches $S_{h}$. However, even when $S_{H}=S_{h}$, the $P_{r}^{(a)}$ obtained using the relatively compliant system leads to noticeable error in the estimates of $S_{H}$ as long as the conventional equation of Eq. (1) is used for the estimation, i.e. the error of $70 \%$ in the example of Fig. 6(a).

It is necessary to beware of the fact that the results of Fig. 7 are functions of parameters assumed in the simulation. For example, there was assumed to be a pair of 1-m high fractures crossing a borehole with an internal diameter of $100 \mathrm{~mm}$. The error in $S_{H} *$ will decrease with increas- 


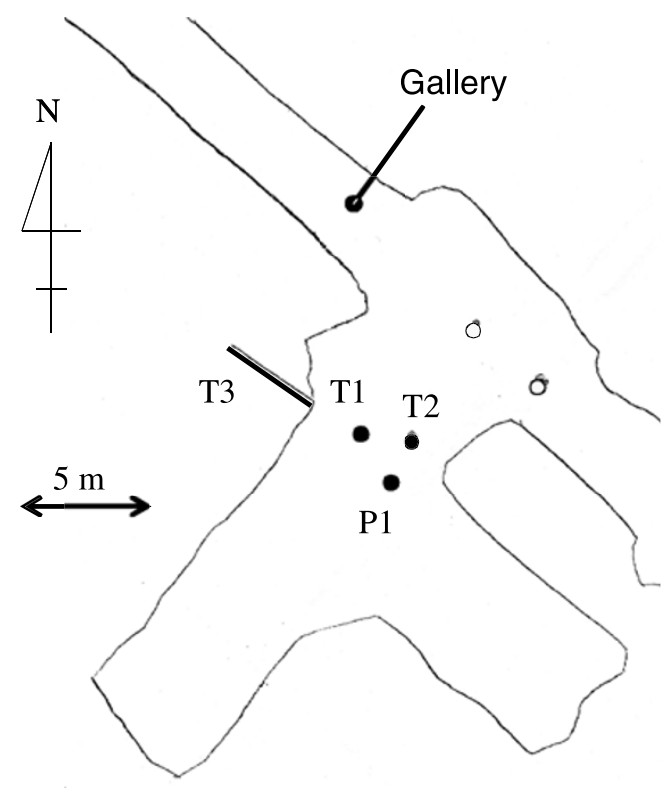

Fig. 8. Plan view of the test site in the Kamaishi mine.

ing fracture height $h$, since the fracture compliance $d V_{c} / d P$ involved in Eq. (4) is in proportion to $h$. The error will increase with increasing borehole size, since the borehole deforms as a result of pressurization easier with increasing borehole size and results in increasing the system compliance $C$ involved in Eq. (4). On the other hand, it is inferred from the numerical analysis of Rutqvist et al. (2000) that if the effect of fracture normal stiffness on the fracture opening cannot be neglected in contrast to the assumption of the present analysis, the fracture opening behavior will be more gradual compared with the results of Fig. 6(b). Such a change may produce some additional error in the detection of $P_{r 0}$ and the estimation of $S_{H}$.

\section{Experimental Verification}

\subsection{Test site and test procedure}

We carried out field tests of hydraulic fracturing to demonstrate the validity of the theoretical considerations on the effect of system compliance. The test site of the Kamaishi iron mine is located in the northeast district of Honshu, Japan's main island. Figure 8 shows a plan view of the experimental site in that mine, at a depth of approximately $300 \mathrm{~m}$ from the ground surface. The major rock of this test site is gnanodiolite. The tests were carried out in two boreholes, ERI04-T1 and ERI04-T2, drilled vertically from the floor of a chamber. ERI04-T1 and ERI04-T2 are hereafter referred to T1 and T2. Both boreholes are $76 \mathrm{~mm}$ in diameter and have a total depth of $20 \mathrm{~m}$. T2 is about $2 \mathrm{~m}$ east of T1.

In this test, we prepared two kinds of hydrofracturing system, i.e. compliant and stiff systems, as illustrated in Fig. 9. For the compliant system, a pump and test interval of a straddle packer were connected with a flexible hydraulic tube which is commonly used for a hydrofracturing system of the wireline type. The tube has an internal diameter of $9.5 \mathrm{~mm}$ and is $200 \mathrm{~m}$ in length. The straddle packer consists of two inflatable rubber packers $630 \mathrm{~mm}$
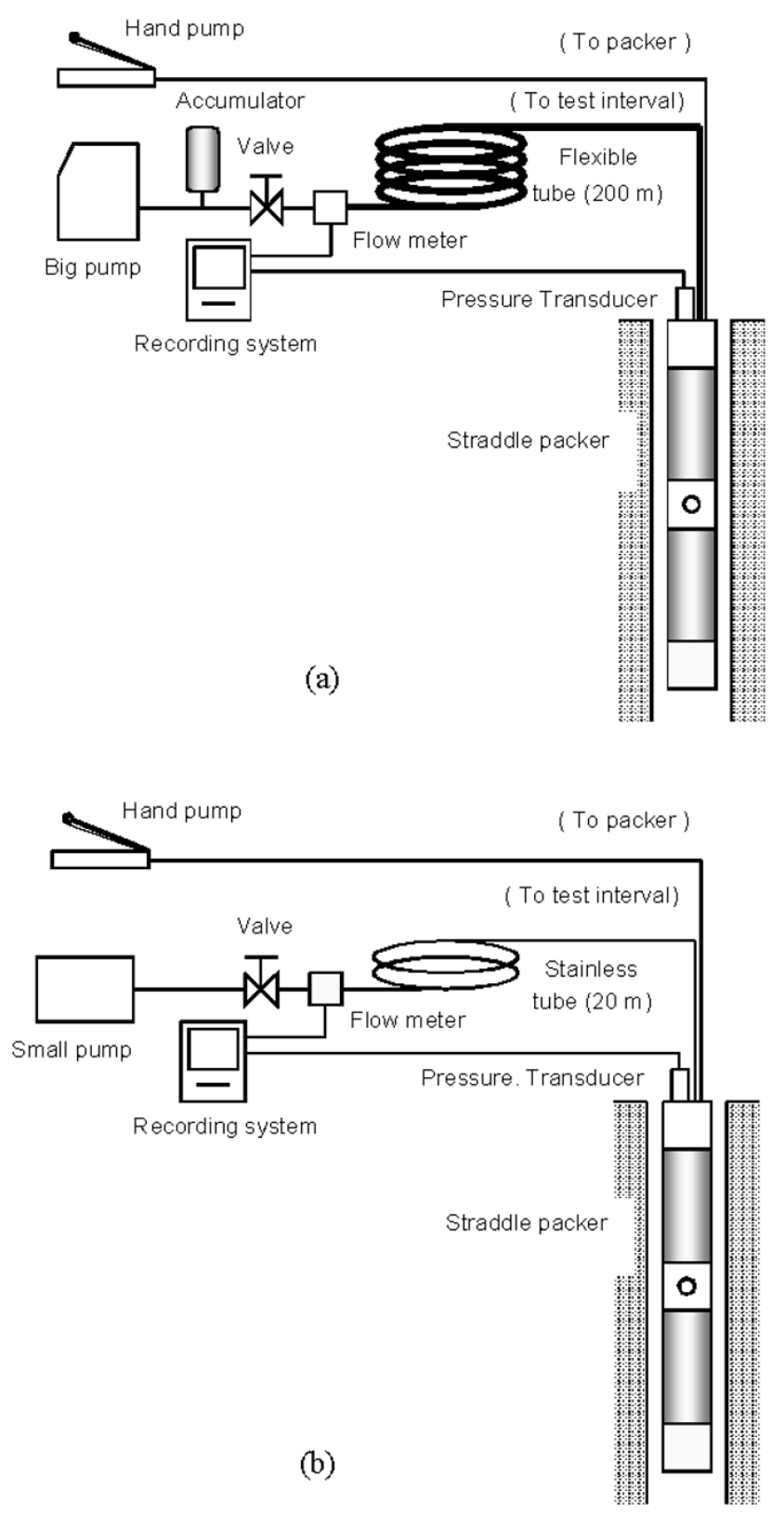

Fig. 9. Two types of test equipment setup used for the present tests. Conventionally compliant system with large compliance (a), and stiff system (b).

in length, and they are spaced apart a distance of $540 \mathrm{~mm}$ so that the length of the test interval is $540 \mathrm{~mm}$. An airdriven pump with a maximum pumping-rate capacity of 2 $1 / \mathrm{min}$ was used to pressurize the test interval, and the pumping rate was measured by a turbine-type flow meter with a measuring range of 1.3 and $13 \mathrm{l} / \mathrm{min}$ (HO-1/2×1/4, Japan Flow Control Co., Japan), which was installed at a place close to the pump. It should be recalled here that the compliance $C$ involved in Eq. (5) is that of the volume between the flow meter and the fracture mouth. Taking this account, for the setup of stiff system, we replaced the flexible hydraulic tube with a seamless stainless steel pipe with an internal diameter of $1.8 \mathrm{~mm}$ and a length of $30 \mathrm{~m}$. According to this modification, the air-driven pump was replaced with the electrically-driven pump with a small pumping-rate capacity of $500 \mathrm{ml} / \mathrm{min}$ at maximum, since the reduced com- 


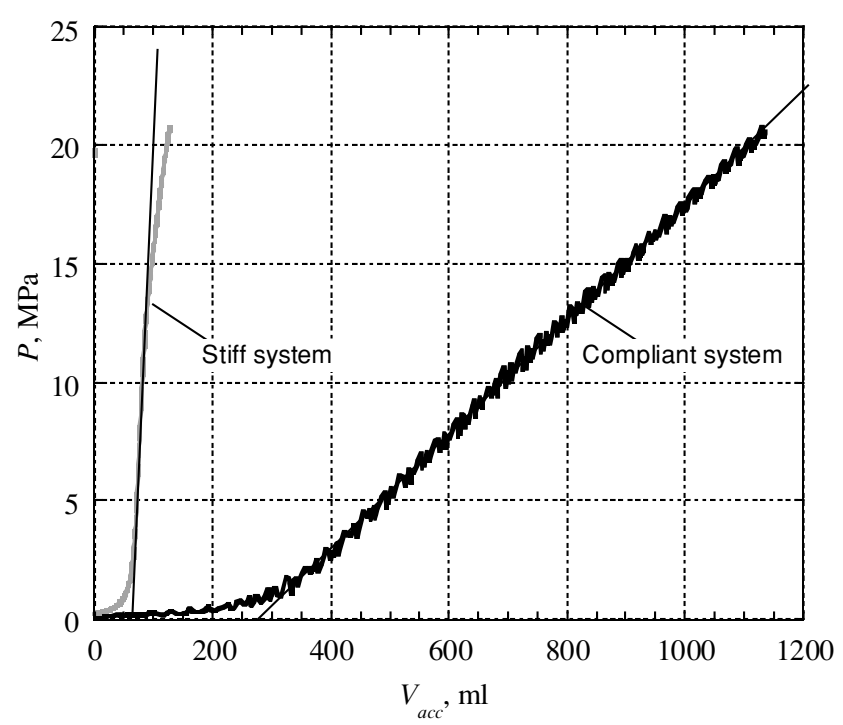

Fig. 10. Plot of pressure versus accumulated volume injected, $V_{\text {acc }}$, observed in tests to measure system compliance of hydrofracturing systems. The tests were carried out in a steel pipe with the same inner diameter as the borehole used for hydrofracturing tests. The compliance can be determined as the inverse of the average inclination of the curves.

pliance contributes to reducing the pumping rate required for the pressure increasing rate in the reopening test to be at the same level as that for the compliant case. The flow meter was also replaced with the same type but with a smaller one (MF30, Japan Flow Control Co.), which is available in the range of 65 and $450 \mathrm{ml} / \mathrm{min}$. The straddle packer was common to these two systems. Figure 10 shows the results of tests in which the compliances of the two systems were measured by pressurizing each of them within a steel pipe which was of the same size as the borehole, where $V_{\text {acc }}$ is the accumulated volume injected. Packer pressure was set to be $12 \mathrm{MPa}$ in the tests. From the viewpoint of reducing system compliance, packer pressure should be set as high as possible to suppress packer deformation. The small fluctuation in the $P-V_{\text {acc }}$ curve for the compliant system was caused by the reciprocating motion of the piston in the airdriven pump, and it was considered not to affect the present tests essentially. The compliance can be estimated as the inverse of the slope of $P-V_{\text {acc }}$ curve. We then found that the compliances are $41.2 \mathrm{ml} / \mathrm{MPa}\left(=4.12 \times 10^{-5} \mathrm{~m}^{3} / \mathrm{MPa}\right)$ and $1.62 \mathrm{ml} / \mathrm{MPa}\left(=1.62 \times 10^{-6} \mathrm{~m}^{3} / \mathrm{MPa}\right)$ for the compliant and stiff systems respectively. Note that the $P-V_{\text {acc }}$ curve obtained using the stiff system deviates slightly from the initial linear trend at the pressure close to the packer pressure of $12 \mathrm{MPa}$; however, such a tendency does not appear on the $P-V_{\text {acc }}$ curve obtained using the compliant system. These results are considered to reveal the effect of system compliance on the variation in borehole pressure.

Using those systems, we carried out the hydrofracturing tests according to the following procedure. First, the straddle packer with the setup of stiff system was lowered to a depth, and the two packers were inflated to isolate a test interval. Packer pressure was set to be $12 \mathrm{MPa}$. The test interval was pressurized by a constant rate of water injection at $400 \mathrm{ml} / \mathrm{min}$ to create a pair of vertical fractures. Frac-
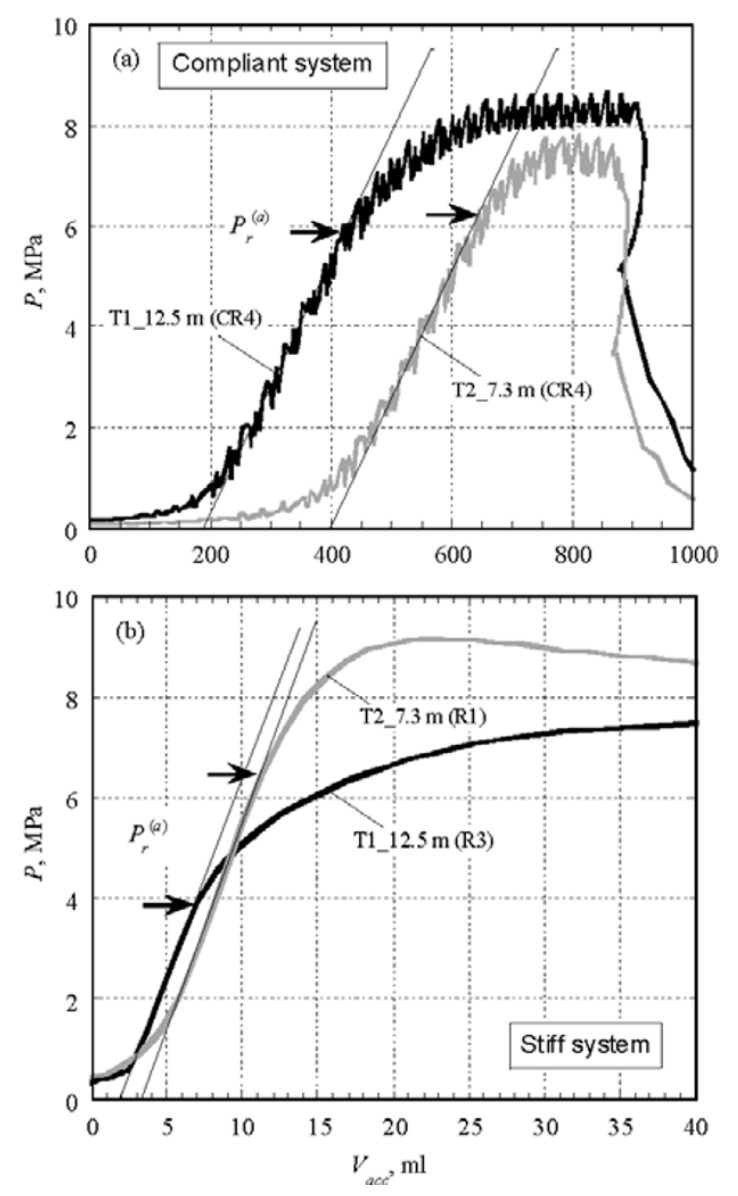

Fig. 11. Plot of pressure versus accumulated volume injected, $V_{\text {acc }}$, observed in fracture opening tests for using the compliant system (a) and the stiff system (b). The points at which the curves deviate from these initial trends define the apparent reopening pressures and are indicated by arrows.

ture size was kept to a minimum by terminating injection as soon as breakdown occurred. Fracture-reopening cycles were conducted a few times at a flow rate in the range of 100 and $400 \mathrm{ml} / \mathrm{min}$. Injection was terminated as soon as the pressure reached a stable level so as to minimize the fracture size and inhibit mismatch of the surfaces, which can promote residual permeability. The fracture was drained after each injection cycle. The packers were then deflated to pull out the straddle packer from the borehole. The system was reset to be the compliant one, and the straddle packer was lowered and set again at the same test interval. Fracturereopening cycles were conducted a few times at a flow rate in the range of 1 and 2 1/min. Finally, an oriented impression packer survey was run to confirm whether the pressurization induced favorably vertical fractures on the borehole wall.

\subsection{Test results}

The hydrofracturing successfully induced vertical fractures at depths of $12.5 \mathrm{~m}$ in T1 and $7.3 \mathrm{~m}$ in T2. We compared the $P-V_{\text {acc }}$ curves of the reopening cycles which were obtained at the injection rate of $Q=1(1 / \mathrm{min})$ using the compliant system and $Q=400(\mathrm{ml} / \mathrm{min})$ using the stiff system. The $P-V_{\text {acc }}$ curves are summarized in Fig. 11(a) and (b) respectively, where the small fluctuation in the $P$ - 
$V_{\text {acc }}$ curves obtained using the compliant system was caused by the reciprocating motion of the piston in the air-driven pump, as noted previously, and its effect on fracture behavior could therefore be ignored. The accumulated injection volume $V_{\text {acc }}$ is used instead of injection time $t$ in those plots, because contrary to numerical simulations, it is hard to maintain a constant injection rate in experiments, and thus the initial pressure rise is not linear with $t$. If the injection rate could be kept constant, which is the ideal situation, the $P-V_{\text {acc }}$ curve should be equal to the $P-t$ curve in shape.

The two $P-V_{\text {acc }}$ curves shown in Fig. 11(a) obtained using the compliant system are similar in shape, while the test locations are different. The two curves rise monotonically and deviate from these trends at pressures of 5.9 and 6.2 $\mathrm{MPa}$ for T1 and T2 respectively, so that the apparent reopening pressures $P_{r}^{(a)}$ are determined as 5.9 and 6.2 $\mathrm{MPa}$ for $\mathrm{T} 1$ and $\mathrm{T} 2$, respectively. Contrary to this, an obvious difference in shape appears between the $P-V_{\text {acc }}$ curves of Fig. 11(b) obtained using the stiff system. Note that the ordinate scale of Fig. 11(b) is the same as that of Fig. 11(a) but that the abscissa scale of Fig. 11(b) is different from that of Fig. 11(a). The $P-V_{\text {acc }}$ curve for T2 deviates from the initial linear trend at a pressure of about $6.4 \mathrm{MPa}$ similarly to the case presented in Fig. 11(a), whereas the $P-V_{\text {acc }}$ curve for $\mathrm{T} 1$ deviates at a pressure of about $3.9 \mathrm{MPa}$, which is clearly lower than the value of 6.4 MPa for T2. On the other hand, the minimum stress $S_{h}$ are estimated from the measured shut-in pressures to be 6.7 and $6.6 \mathrm{MPa}$ for $\mathrm{T} 1$ and $\mathrm{T} 2$, respectively. Thus, although the shut-in pressure is almost of the same magnitude, the reopening pressure obtained using the stiff system is distinctly different for $\mathrm{T} 1$ and $\mathrm{T} 2$. This indicates that the reopening pressure may show some sensitivity to the maximum stress $S_{H}$ in the case using the stiff system, although because of unknown in-situ stresses, it is hard to confirm whether the detected reopening pressures are related to in-situ stresses following Eq. (4). Furthermore, we were able to presume the validity of our simulations from the similarities between the observed $P$ $V_{\text {acc }}$ curves of Fig. 11 and the simulated $P$ - $t$ curves of Fig. 6. Namely, the $P_{r}^{(a)}$ obtained using the stiff system is equal to or lower than that obtained using the compliant system, and when the $P_{r}^{(a)}$ obtained using the stiff and compliant systems are the same, a peak of the $P$ - $t$ curve obtained using the stiff system is higher than that obtained using the compliant system.

It is assumed here that the compliance of the stiff system is small enough for the detection of the true reopening pressure. Then, from Eq. (7) and the values of $P_{r}^{(a)}$ obtained using the stiff system-i.e. 3.9 and 6.4 MPa for T1 and T2, respectively-the maximum stress $S_{H}$ are estimated to be 12.3 and $7 \mathrm{MPa}$ for T1 and T2, respectively. Thus, the $S_{h}$ are almost the same as those described above, but the $S_{H}$ are fairly different at these two test locations, even though the locations are less than $10 \mathrm{~m}$ apart. Such a complicated stress distribution may be caused by the fact that the $S_{H}$ at the test location in T2 was relaxed by movement of a fault dipping roughly to the $S_{H}$ azimuth, where such a fault was actually found about $1 \mathrm{~m}$ below the test location in T2. Due to the stress relaxation, the stress state at the test location in
T2 may have become hydrostatic. This idea is supported by the fact that the fracture orientation indicating the $S_{H}$ azimuth at the test location of $\mathrm{T} 2$ is rotated $44^{\circ}$ to east from that at the test location of $\mathrm{T} 1$, i.e. $\mathrm{N}^{\circ} \mathrm{E}$.

\section{Conclusions}

With the aid of numerical simulation, we examined how the residual aperture affects pressure distribution with respect to fracture and pressure variation in a borehole during the hydrofracturing test. The results show that, despite assuming the small residual aperture of $2 \mu \mathrm{m}$, residual aperture causes significant pressure penetration into the fracture. The pressure distributes uniformly, becoming almost equal to the borehole pressure $P$ already at the moment of fracture opening. The pressure penetration into the fracture prior to opening is to reduce the borehole pressure at the moment of fracture opening, $P_{r 0}$, by a factor of two from the value expected using the conventional theory, whereas $P_{r 0}$ is still a function of the maximum horizontal stress $S_{H}$.

After the borehole pressure is beyond $P_{r 0}$, the fracture begins to open. However, due to the stress concentration around the borehole, the open portion of fracture is limited initially in the vicinity of the borehole, and because of such a small change in the fracture volume, no detectable change occurs on the $P$ - $t$ curve, where $t$ is injection time. With increasing $P$, the stress concentration in compression is weakened gradually, and when $P$ reaches a level of the minimum stress $S_{h}$, the fracture aperture increases abruptly with small increments in borehole pressure; in other words, the fracture compliance increases abruptly. The $P$ - $t$ curve then begins to deviate from the initial linear trend so that an apparent reopening pressure $P_{r}^{(a)}$ appears finally on the $P$ - $t$ curve. The same process occurs irregardless of the maximum stress $S_{H}$. This process provides an explanation as to why there are so many incidences where the apparent reopening pressure coincides with the shut-in pressure; in other words, why incidences of the minimum stress $S_{h}$ are so common in the literature.

The discrepancy that arises between $P_{r 0}$ and $P_{r}^{(a)}$ because of the compliance of typical hydraulic fracturing systems is far larger than that of the fracture until $P$ reaches a level of the minimum stress $S_{h}$. Even if a flexible hydraulic tube with small internal diameter of less than $10 \mathrm{~mm}$ is used to convey fracturing fluid from a pump to a test section in a borehole, the system compliance $C$ is so large that $P_{r}^{(a)}$ does not coincide with $P_{r 0}$. For the stress measurements carried out to date at depths beyond $500 \mathrm{~m}$, drill pipes have been used more commonly rather than the flexible hydraulic tube. The drill pipe usually has an inner diameter of more than $50 \mathrm{~mm}$, and such a size is enough to expect fairly large system compliance compared with the case in which the flexible tube is used. This should alert us to the fact that a large error is included in the estimates of maximum stress $S_{H}$ based on the reopening pressure data collected to date.

On the other hand, our model shows that $P_{r}^{(a)}$ varies as a function of $C$. Such a feature of $P_{r}^{(a)}$ was demonstrated by field experiments carried out at the Kamaishi iron mine in Japan. If the $C$ can be reduced small enough, then $P_{r}^{(a)}$ approaches the true reopening pressure $P_{r 0}$, so that we could measure $P_{r 0}$ approximately from $P_{r}^{(a)}$, which is detected us- 
ing the stiff hydrofracturing system. The detected $P_{r 0}$ allows us to estimate $S_{H}$ on the condition that the magnitude of $S_{h}$ is known from the shut-in pressure.

The compliance in which this article has focused on is that of the volume between the flow meter and the fracture mouth. The easiest way that this can be reduced is to place the flow meter as close as possible to the test interval so that a more objective measure of flow entering the fracture can be obtained. For tests conducted at depths of more than tens of meters, this practically demands the use of downhole flow meters. In this case, it is not important what kind of tubing-i.e. drill pipe, flexible tube or stainless pipe with a small internal diameter-is used to convey the fracturing fluid from a pump to a test interval.

Acknowledgments. We thank Prof. Y. Mizuta and Prof. J. Rutqvist for their reviews of this manuscript. The authors wish to thank the kind support provided by the Nittetsu Mining Co. Ltd. and Kamaishi Mining Co. Ltd.

\section{References}

Cheung, L. S. and B. C. Haimson, Laboratory study of hydraulic fracturing pressure data-How valid is their conventional interpretation?, Int. J. Rock Mech. Min. Sci. Geomech. Abstr., 26, 595-604, 1989.

Cornet, F. H., Analysis of injection tests for in-situ stress determination,
Proc. Workshop Hydraulic fracturing Stress Measurement, Menlo Park, 414-443, 1982.

Durham, W. B. and B. P. Bonner, Self-propping and fluid flow in slightly offset joints at high effective pressures, J. Geophys. Res., 99, 93919399, 1994.

Evans, K. F., T. Engelder, and R. A. Plumb, Appalachian stress study 1. A detailed description of in-situ stress variations in Devonian shales of the Appalachian Plateau, J. Geophys. Res., 94, 7129-7154, 1989.

Ito, T. and K. Hayashi, Relation between reopening pressure and tectonic stresses for hydraulic fracturing tectonic stress measurements, Proc. 8th Int. Cong. Computer Methods and Advances in Geomech., Morgantown, 1591-1596, 1994.

Ito, T., K. Evans, K. Kawai, and K. Hayashi, Hydraulic fracture reopening pressure and the estimation of maximum horizontal stress, Int. J. Rock Mech. Min. Sci. Geomech. Abstr., 36, 811-826, 1999.

Lee, M. Y. and B. C. Haimson, Statistical evaluation of hydraulic fracturing stress measurement parameters, Int. J. Rock Mech. Min. Sci. Geomech. Abstr., 26, 447-456, 1989.

Rutqvist, J., C.-F. Tsang, and O. Stephansson, Uncertainty in the maximum principal stress estimated from hydraulic fracturing measurements due to the presence of the induced fracture, Int. J. Rock Mech. Min. Sci. Geomech. Abstr., 37, 107-129, 2000.

Zoback, M. D., F. Rummel, R. Jung, and C. B. Raleigh, Laboratory hydraulic fracturing experiments in intact and pre-fractured rock, Int. J. Rock Mech. Min. Sci. Geomech. Abstr., 14, 49-58, 1977.

T. Ito (e-mail: ito@ifs.tohoku.ac.jp), A. Igarashi, H. Kato, H. Ito, and O. Sano 